\title{
Foreword
}

This volume documents the morphology, taxonomy and diversity of coccolithophores in the North Western Mediterranean. Coccolithophores are minute organisms but they are a key component of the oceanic ecosystem and play a major role in the global carbon cycle. So they have attracted multi-disciplinary interest and been intensively studied over the past decade, notably through the European EHUX and CODENET projects. Particularly within the CODENET (Coccolithophorid Evolutionary Biodiversity and Ecology Network) project we recognised the importance of biodiversity in determining the responses and impacts of coccolithophores on global change processes. So, whilst our prime focus was on key major species, we were very pleased to be able to encourage within the project collaboration between taxonomic enthusiasts to develop knowledge of their diversity. This atlas is one spectacular outcome with a fine combination of scientific value and graphic beauty. It is also testimony to an immense investment of time and effort.

Cocccolithophores are remarkably small, only a few microns long and a micron is one millionth of a meter. The typical scale of the plates in this volume is 10,000:1 at which scale a micron is one centimetre long, and a centimetre would be one hundred metres long. One technique I like for understanding the scale is to look out of the window, visualise one hundred metres, mentally enlarge a fingertip to that scale, then look back at a picture of a coccolithophore. As this thought experiment shows the scanning electron microscope achieves quite extraordinary magnifications. It also produces very beautiful images of surface topology and topography which are directly comprehensible. SEMs are not, however, the fastest of machines to use - working on the SEM entails sitting for long hours in a darkened room staring at a grainy low resolution image on a small monitor counting specimens and watching out for exceptional specimens. Photographing specimens requires careful alignment, destigmatisation and focussing of the electron beam then a long wait as the beam is slowly scanned across the specimen building up the image. A dozen images in an hour is a good rate, if one can find nice specimens and the machine is working well. The images in this atlas represent thousands of man hours of work over a six year period. Their quality reflects a highly productive and very friendly, long-term collaboration between Lluisa Cros and José-Manuel Fortuño, who has produced outstanding micrographs from a rather normal SEM.

The text, which is entirely Lluisa's work, progresses the Atlas from illustration to full scientific description. It is the first monograph of coccolithophores for a decade and the first ever with such comprehensive coverage. It also contains some remarkable data. At least three aspects should be noted - first the major revision of the most problematic single genus, Syracosphaera, based on a wealth of new observations of exothecal coccoliths, which had never previously been studied systematically. Second, the documentation of numerous combination coccospheres capturing the transition between haploid and diploid life-cycle phases and enabling integration of the previously independent taxonomies of these two phases. The first of these SEMs were available as the CODENET project commenced and they stimulated wide-ranging work on coccolithophorid life-cycles. Others were found as the project continued including the remarkable combinations of Alisphaera and Canistrolithus with Polycrater (Figures 77-79) printed here for the first time. Third, the documentation of previously unsuspected biodiversity. There are about 50 informally described species in this monograph which have not been recorded previously, and several more which have been formally described as result of this study, notably two with names 
of special significance: Alisphaera gaudii commemorating Barcelona's most famous architect and Picarola margalefii commemorating Ramon Margalef, who has a comparable status among marine ecologists and who inspired and directed this study. In a group supposedly as well-studied as the coccolithophores this many new species is remarkable and unexpected. It reflects painstaking study of the smaller forms, study of a very large number of specimens and a fine eye for detail leading to separation of superficially similar species. It also perhaps reflects some special conditions of the NW Mediterranean where a mix of Mediterranean and Atlantic waters creates variable conditions stimulating biodiversity. In any event this monograph will both be an invaluable reference for anyone studying Mediterranean plankton and a milestone in the development of taxonomy of the coccolithophores.

\section{JEREMY YOUNG}

Head of Micropalaeontology,

The Natural History Museum, London

and co-ordinator of the CODENET project 